\title{
Simulation of long-streamer propagation in air at atmospheric pressure
}

\author{
N L Aleksandrov $\dagger$ and E M Bazelyan $\ddagger$ \\ $\dagger$ Department of Physical Mechanics, Moscow Institute of Physics and Technology, \\ 141700 Dolgoprudny, Russia \\ ¥ Krzhizhanovsky Power Engineering Institute, 117927 Moscow, Russia
}

Received 9 June 1995, in final form 10 October 1995

\begin{abstract}
The propagation of the long cathode-directed streamer in air is simulated in the approximation of a fixed radius of the streamer channel. The results of the simulation turn out to be sensitive to the channel radius and to a model of charged particle kinetics. A model including ionization expansion of the streamer channel and yielding results which depend only slightly on the initial channel radius is developed. For the streamer's propagation in a non-uniform field, our calculation agrees well with the available experimental values of the total anode current, the streamer length and the average electric field in the streamer channel. Simulation of the streamer's evolution in a uniform electric field shows a steady propagation if the field exceeds $8-8.5 \mathrm{kV} \mathrm{cm}^{-1}$.
\end{abstract}

\section{Introduction}

Streamer propagation is an important discharge phase of the breakdown of a long gas gap. In electropositive gases, such as Ar, a streamer bridging a gap tens of centimetres in length can immediately transform into an arc channel [1]. In electronegative gases, such as air, the tip of a leader advancing toward the opposite electrode is preceded by a cloud of streamers [2]. These streamers arise with a frequency up to $10^{9}-10^{10} \mathrm{~s}^{-1}$ to supply the channel of a long spark or lightning with electric current and power. The streamer zone can exceed $10 \mathrm{~m}$ in length. It is impossible to develop a consistent theory of long sparks in gases without examination of the long-streamer propagation and the properties of the streamer plasmas which considerably vary in space and time. Nowadays the interest in these transient filamentary plasmas is due to their application to the removal of air-borne toxic chemicals [3-5]. In addition, there is reason to believe that the streamers in liquid media are of a gaseous nature [6].

It is hard to carry out any experiment with long streamers since high temporal resolution $\left(10^{-9} \mathrm{~s}\right)$ and spatial resolution $\left(10^{-2}-10^{-3} \mathrm{~cm}\right)$ are required; it is difficult to predict the streamer path and to observe the streamer because of the low intensity of the light emitted by the streamer channel. In addition, it is difficult to use sensitive equipment when high impulse voltages (up to $10^{6} \mathrm{~V}$ ) are applied to generate long streamers. For this reason the reliable data in the current literature cover only some averaged characteristics of the long streamers, such as the ignition voltage, the propagation velocity in various electric fields and their pulse-rise-rate conditions, the anode conduction current, the net space charge and the charge of the head (the forward streamer domain of the size of the channel radius) of the isolated streamer and the averaged longitudinal electric field in its channel. Properties of the long-streamer plasmas are poorly understood. Some efforts have been made to estimate the gas temperature of the streamer channel, the time-averaged density of electrons and the conductivity of the channel per unit length. (The conductivity per unit cross section remains unknown because the channel radius is still unmeasured.) The reviews of the experimental long-streamer studies [711] indicate that the available information is still insufficient not only to solve applied problems, but also to determine input parameters for simulating streamers numerically and testing the developed theories.

Thus, it is no wonder that a great deal of effort has been devoted to computer simulation of the streamer-plasma properties [11,12]. It is important to bear in mind that a consistent theoretical model of a long streamer should be two-dimensional (2D). Moreover, in most cases the streamer propagation appears to be a three-dimensional (3D) phenomenon due to discharge branching or an interaction between streamers which start concurrently from the high-voltage electrode or the leader tip. The development of the 3D long-streamer models is currently open to question; therefore, simpler approaches have been used to study the streamer dynamics.

The majority of the 2D calculations are devoted to investigation of the avalanche-to-streamer transition [1316] or to the formation and propagation of the short 
streamers (about $1-5 \mathrm{~cm}$ in length) [17-22]. The simulation of streamers is generally based on a representation of a space-charge filament by a series of charged discs of equal radii, with uniform densities of space charge and of other plasma parameters over each disc (the $1.5 \mathrm{D}$ model) [2325]. The radius is supposed to be equal to that of the initial electron avalanche $(0.003-0.01 \mathrm{~cm})[23,24]$. The method of discs allows one to calculate the value of the electric field on the streamer axis. The dynamic behaviour of electron and ion assemblies in a background gas, as a rule, is described by means of the drift-diffusion approximation because comparison of Monte Carlo and fluid equation treatments of streamer dynamics has demonstrated the validity of the fluid treatment for modelling short streamers $[18,20]$.

A little attention has been given to the dependence of the results of numerical simulation on the a priori given channel radius and on the rate coefficients of electronand ion-molecule processes. The streamer plasma in air is generally assumed to be composed of electrons and onespecies positive and negative ions; therefore, the averaged rate coefficients for ionization, electron attachment and detachment, electron-ion and ion-ion recombination are used. However, a description of the kinetics of a gas discharge in air considered as an oxygen-nitrogen mixture can include more than 40 reacting constituents and more than 250 reactions [26]. It should be noted that the number of processes required for the simulation increases with the streamer length because of an increase in the lifetime of the streamer plasma.

Using a $1.5 \mathrm{D}$ code, we recently showed that a variety of electron- and ion-molecule processes plays an important role for the dynamics of the long cathode-directed streamer $(50-100 \mathrm{~cm})$ in air [11]. In addition, a strong dependence of the results of simulation on the fixed streamer-channel radius has been observed. This has cast doubt on the possibility of obtaining quantitative data on the longstreamer properties by means of the $1.5 \mathrm{D}$ modelling, that is, the fixed-channel-radius approximation. There is also reason to think that this approach fails to describe the behaviour of long streamers because of the expansion of the streamer channel resulting from the ionization in the radial electric field of the streamer [11,27, 28].

In the present paper, we generalize our streamersimulation approach $[11,27,28]$ in order to take into account the ionization expansion of the streamer channel and, therefore, a change in the channel radius along the streamer. A cathode-directed streamer in the air gap between a spherical anode and an infinitely distant cathode is studied. In section 2, our $1.5 \mathrm{D}$ model is considered and some results of the streamer simulation in the fixed-radius approximation are given. The model accounting for the channel expansion is discussed in section 3. The results of our calculations in this case in non-uniform and uniform electric fields are presented in sections 4 and 5, respectively. A comparison with experimental data is also given there.

\section{The model of a streamer with fixed channel radius. Foundations and some results}

We consider a cathode-directed streamer which is of great applied interest and is under active experimental study. The basic dynamical equations for the streamer propagation are the continuity equations for electrons, ions and active particles, and Poisson's equation for the electric field:

$$
\begin{aligned}
\frac{\partial n_{e}}{\partial t} & +\nabla \cdot\left(n_{e} \boldsymbol{v}_{e}-\mathbf{D}_{e} \nabla n_{e}\right)=\left(k_{i} N+k_{i}^{*} n^{*}\right) n_{e} \\
& -\left(k_{a}^{\prime}+k_{a}^{\prime \prime} N\right) N_{a} n_{e}+\left(k_{d} N+k_{d}^{*} n^{*}\right) n_{n}-\beta_{e i} n_{e} n_{p}+S_{f}
\end{aligned}
$$

$\frac{\partial n_{p}}{\partial t}+\nabla \cdot\left(n_{p} \boldsymbol{v}_{p}\right)=\left(k_{i} N+k_{i}^{*} n^{*}\right) n_{e}-\beta_{e i} n_{e} n_{p}$ $-\beta_{i i} n_{p} n_{n}+S_{f}$

$\frac{\partial n_{n}}{\partial t}+\nabla \cdot\left(n_{n} \boldsymbol{v}_{n}\right)=\left(k_{a}^{\prime}+k_{a}^{\prime \prime} N\right) N_{a} n_{e}-\left(k_{d} N+k_{d}^{*} n^{*}\right) n_{n}$ $-\beta_{i i} n_{p} n_{n}$

$\frac{\partial n^{*}}{\partial t}=k^{*} N n_{e}-k_{i}^{*} n^{*} n_{e}-k_{q}^{*} N n^{*}-\frac{n^{*}}{t_{l}}$

$\boldsymbol{\nabla} \cdot \boldsymbol{E}=\frac{e}{\varepsilon_{0}}\left(n_{p}-n_{e}-n_{n}\right)$.

Here $n_{e}, n_{p}, n_{n}$ and $n^{*}$ are the electron, positive-ion, negative-ion and active-particle densities, respectively, $\boldsymbol{v}_{e}$, $\boldsymbol{v}_{p}$ and $\boldsymbol{v}_{n}$ are the corresponding drift velocities, $\mathbf{D}_{e}$ is the electron diffusion tensor, $\boldsymbol{E}$ is the electric field vector, $e$ is the electric charge, $\varepsilon_{0}$ is the permittivity of free space, $k_{i}, k_{i}^{*}, k_{a}^{\prime}, k_{a}^{\prime \prime}, k_{d}, k_{d}^{*}, \beta_{e i}, \beta_{i i}, k^{*}$ and $k_{q}^{*}$ are the rate coefficients for direct and stepwise ionization, dissociative and three-body electron attachment, electron detachment in collisions with ground-state molecules and active particles, electron-ion and ion-ion recombinations, and formation and loss of active particles, respectively; $N$ and $N_{a}$ are the total density of neutral species and the electronegative-component density and $t_{l}$ is the lifetime of an excited molecule. $S_{f}$ describes the photo-ionization source. Following previous studies [17,21], we neglect photo-ionization and assume that our streamers propagate through a pre-existing low-ionization air plasma with $n_{e}(t=0)=10^{8} \mathrm{~cm}^{-3}$. In our model, ion diffusion is assumed to be negligible for cold ions. The drift velocities and the rate coefficients are functions of the local reduced electric field $E / N$ and the vibrational temperature of nitrogen molecules, $T_{v}$, which obeys the equation of the vibrational energy balance.

Equations (1)-(4) were solved numerically, a finitedifference method with an adapted mesh was used; the mesh was stretched in the axial coordinate near the anode (in the domain of the size of the anode radius) and in the streamer head, and was uniform in other regions. Contrary to the disc method, the space charge was assumed to be evenly distributed over the cylinder channel surface to determine the electric field. This approach is based on the short time $\left(10^{-10}-10^{-11} \mathrm{~s}\right)$ of the streamer-plasma's polarization by the radial electric field. Our so-called ring method gives a simpler expression for the axial electric field:

$E(x)=\frac{\tau_{c}}{4 \pi \varepsilon_{0}}\left\{\left[\left(x_{2}-x\right)^{2}+r_{c}^{2}\right]^{-1 / 2}-\left[\left(x_{1}-x\right)^{2}+r_{c}^{2}\right]^{-1 / 2}\right\}$ 


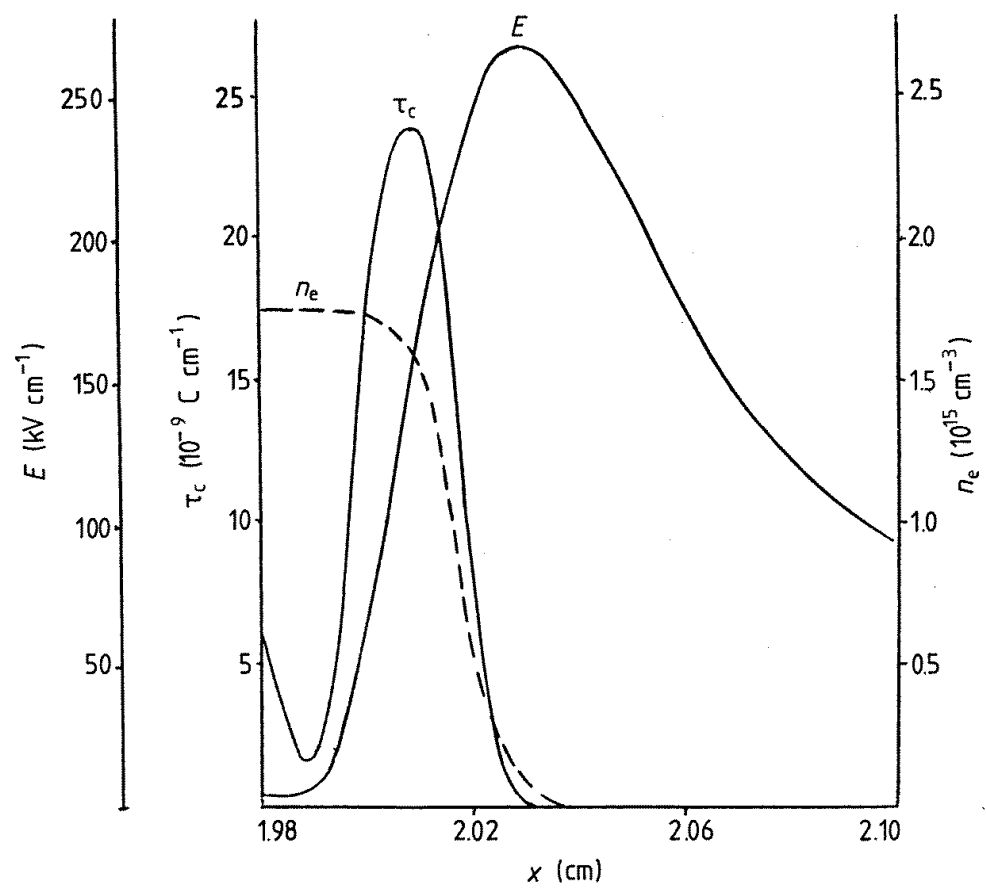

Figure 1. Axial profiles of $E, \tau_{c}$ and $n_{e}$ at the streamer head at $3.95 \mathrm{~ns}$. The initial conditions used are a spherical anode of radius $R_{a}=1 \mathrm{~cm}$, the applied voltage of $100 \mathrm{kV}$ and the fixed radius $r_{c}=0.03 \mathrm{~cm}$.

where $\tau_{c}$ is the channel charge per unit length, and $x_{1}$ and $x_{2}$ are the boundary coordinates of the channel section with the radius $r_{c}$. This markedly decreases the computercalculation time, and, which is more important, prevents the appearance of numerical instabilities. Our assumption concerning the space-charge distribution seems to be true for all streamer regions but that at the streamer head.

The air was assumed to be a $\mathrm{N}_{2}-\mathrm{O}_{2}$ mixture at standard pressure and temperature. We considered 20 components presented by table 1 and 120 collisional processes which are important in short time intervals (about $10^{-6} \mathrm{~s}$ ) with rate coefficients found experimentally or calculated from the Boltzmann equation for electrons [26]. These processes include direct ionization of $\mathrm{N}_{2}$ and $\mathrm{O}_{2}$, two-step ionization through $\mathrm{N}_{2}\left(\mathrm{~A}^{3} \Sigma_{u}^{+}\right), \mathrm{N}_{2}\left(\mathrm{~B}^{3} \Pi_{g}\right), \quad \mathrm{N}_{2}\left(\mathrm{a}^{\prime 1} \Sigma_{u}^{-}\right)$, and $\mathrm{N}_{2}\left(\mathrm{C}^{3} \Pi_{u}\right)$, associative ionization with $\mathrm{N}_{2}\left(\mathrm{a}^{\prime}{ }^{1} \Sigma_{u}^{-}\right)$ and $\mathrm{N}_{2}\left(\mathrm{~A}^{3} \Sigma_{u}^{+}\right)$, three-body electron attachment to $\mathrm{O}_{2}$, dissociative attachment to $\mathrm{O}_{2}$ and $\mathrm{O}_{3}$, electron-ion and ionion recombinations, electron detachment from negative ions in collisions with $\mathrm{O}, \mathrm{N}_{2}\left(\mathrm{~A}^{3} \Sigma_{u}^{+}\right), \mathrm{N}_{2}\left(\mathrm{~B}^{3} \Pi_{g}\right), \mathrm{O}_{2}\left(\mathrm{a}^{1} \Delta_{g}\right)$ and $\mathrm{O}_{2}\left(\mathrm{~b}^{1} \Sigma_{g}^{+}\right)$, respectively, which are excited by electron impact, and so on.

To account for the influence of vibrational and gas temperatures on the electron rate coefficients in air [27, 28] we solved numerically the balance equations for vibrational energy of $\mathrm{N}_{2}$ molecules

$$
\frac{\partial \varepsilon_{v}}{\partial t}=\lambda_{v} j E-Q_{V T} \quad Q_{V T}=\frac{\varepsilon_{v}-\varepsilon_{0}(T)}{\tau_{V T}(T)}
$$

and for the translational energy of neutral species

$$
c_{V} N \frac{\partial T}{\partial t}=\left(\lambda_{T}+\lambda_{R}\right) j E+Q_{V T}+Q_{e T}+Q_{r}+Q_{c} .
$$

Here $\varepsilon_{v}$ is the vibrational energy of $\mathrm{N}_{2}$ molecules, $\varepsilon_{0}(T)$ is the vibrational energy corresponding to the Boltzmann equilibrium at the temperature $T, \lambda_{v}, \lambda_{T}$ and $\lambda_{R}$ are the fractional powers transferred by electrons to the molecules in vibrational excitation, elastic collisions and rotational excitation, respectively, $j$ is the density of the electron current, $\tau_{V T}$ is the time of vibrational relaxation, $c_{V}$ is the heat capacity per molecule at constant volume, $Q_{V T}$ is the contribution from quenching of vibrational levels of $\mathrm{N}_{2}$ in the process of $\mathrm{V}-\mathrm{T}$ relaxation to heat, $Q_{e T}, Q_{r}$ and $Q_{c}$ are the contributions from quenching electronically excited molecules by electrons, from the electron-ion recombination and from the ion conversion, respectively. The details of solving the energy-balance equations and some results for the streamer plasma have been given elsewhere [27, 28]. Gas heating in the streamer channel can usually be neglected whereas the vibrational temperature can attain 2000-3000 K.

In our simulation the time resolution determined by the fastest electron process was down to $10^{-12} \mathrm{~s}$. A space mesh was adopted to resolve the steep profiles of electric field and electron density at the streamer head distinctly, as is shown in figure 1. A disadvantage of any $1.5 \mathrm{D}$ simulation of a long streamer is that the obtained results strongly depend on the unknown radius of the channel. Figure 2 demonstrates the peak values of the streamer velocity, electric field and electron density at the head of the streamer and the conduction current through the anode calculated in our work for a spherical anode of $R_{a}=1 \mathrm{~cm}$ radius and the applied voltage of $100 \mathrm{kV}$. The peak electron density is the most strongly radius-dependent quantity; thus more than a tenfold decrease in the electron density results from a tenfold increase in the radius. 
Table 1. Relevant species contained in the chemistry code.

\begin{tabular}{llllll}
\hline $\mathrm{e}$ & $\mathrm{N}_{2}^{+}$ & $\mathrm{N}_{4}^{+}$ & $\mathrm{O}_{2}^{+}$ & $\mathrm{O}_{4}^{+}$ & $\mathrm{O}_{2}^{+} \mathrm{N}_{2}$ \\
$\mathrm{O}^{-}$ & $\mathrm{O}_{2}^{-}$ & $\mathrm{O}_{3}^{-}$ & $\mathrm{O}_{4}^{-}$ & $\mathrm{O}_{2}$ & $\mathrm{~N}_{2}$ \\
$\mathrm{O}$ & $\mathrm{O}_{3}$ & $\mathrm{~N}_{2}\left(\mathrm{~A}^{3} \Sigma_{u}^{+}\right)$ & $\mathrm{N}_{2}\left(\mathrm{~B}^{3} \Pi_{g}\right)$ & $\mathrm{N}_{2}\left(\mathrm{a}^{\prime}{ }^{1} \Sigma_{u}^{-}\right)$ & $\mathrm{N}_{2}\left(\mathrm{C}^{3} \Pi_{u}\right)$ \\
$\mathrm{O}_{2}\left(\mathrm{a}^{1} \Delta_{g}\right)$ & $\mathrm{O}_{2}\left(\mathrm{~b}^{1} \Sigma_{g}^{+}\right)$ & $\mathrm{O}\left({ }^{1} \mathrm{D}\right)$ & $\mathrm{O}\left({ }^{1} \mathrm{~S}\right)$ & & \\
\hline
\end{tabular}

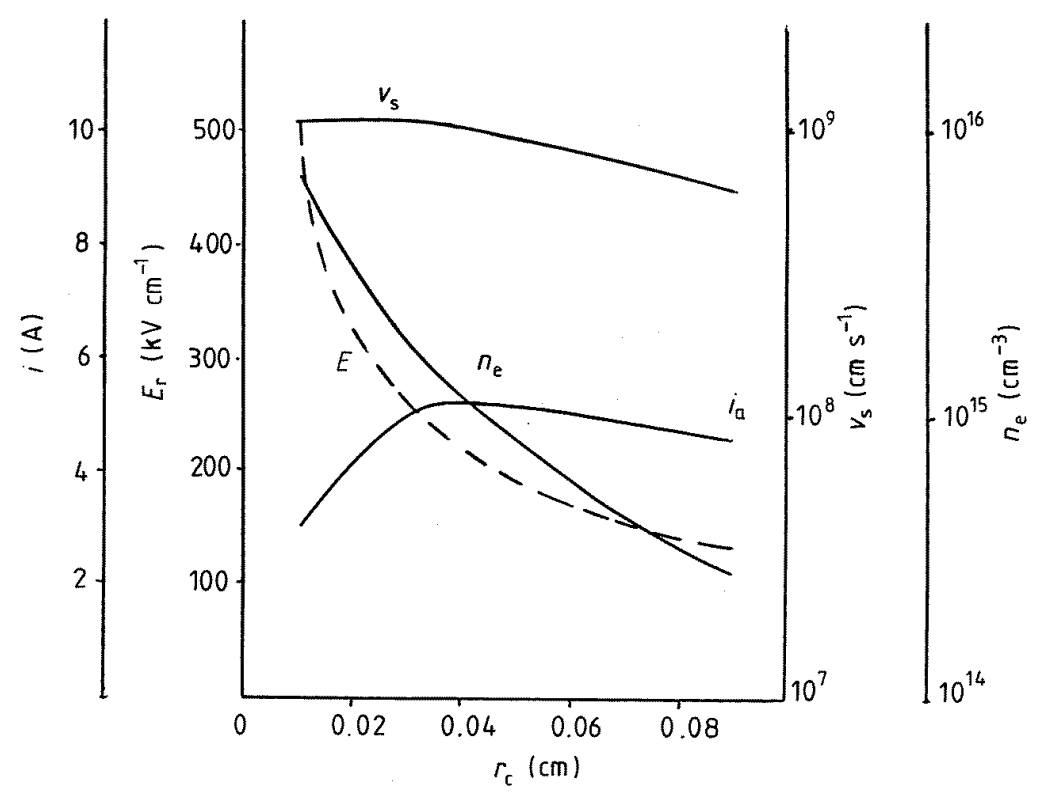

Figure 2. The peak values of the streamer velocity, electric field and electron density at the streamer head, and conduction current through the anode versus $r_{c}$. The curves correspond to the same initial conditions as those given in figure 1 .

One can see that the inverse relation between electron density in the wavefront, $n_{e f}$, and the head (or channel) radius, $r_{c}$, is an inherent feature of any ionization wave propagating due to ionization in the front electric field. A steady propagation of the wave with velocity $v_{s}$ results from re-induction of the front electric field $E_{m}$; to do this, the density of electrons generated in the front region must be sufficiently high to create the head charge $g_{h} \simeq 4 \pi \varepsilon_{0} r_{c}^{2} E_{m}$ for a time $\Delta t \simeq r_{c} / v_{s}$. The charge is induced owing to the drift of electrons with the electric current $i_{h} \simeq$ $\pi r_{c}^{2} e n_{e f} \mu_{e} E_{m}$, where $\mu_{e}$ is the electron mobility. Equating the current to $g_{h} / \Delta t$ we find the electron density in the wavefront to be

$$
n_{e f} \simeq \frac{4 \varepsilon_{0} v_{s}}{\mathrm{e} \mu_{e} r_{c}} .
$$

The inverse relation between $n_{e f}$ and $r_{c}$ agrees well with our numerical results shown in figure 2 .

Though suitable for a qualitative analysis, the $1.5 \mathrm{D}$ model fails to describe some experimental data. A number of experiments has been performed to study an impulse corona in air and to measure the total streamer current through the spherical anode with $R_{a}=12.5 \mathrm{~cm}$ at the initial electric field of $40 \mathrm{kV} \mathrm{cm}^{-1}$ near the anode $[8,29]$. Under these conditions the current ranges up to about $10 \mathrm{~A}$ without streamer branching. Using the $1.5 \mathrm{D}$ simulation we have calculated the total anode current which was well below $10 \mathrm{~A}$. An increase in the channel radius to $0.2 \mathrm{~cm}$ results in a current of no more than $2 \mathrm{~A}$. With further increase in the radius, the current does not increase because of a sharp fall of the electric field in front of the streamer head.

In $1.5 \mathrm{D}$ modelling, the radius of the streamer channel is usually assumed to be equal to that of the initial electron avalanche. This is not valid because of the ionization in the radial electric field produced by a high channel charge. Near the surface of a long channel this field is given by

$$
E_{r}(x) \simeq \frac{U_{c}(x)}{r_{c} \ln \left(l / r_{c}\right)}
$$

when $E_{c}(x) r_{c} \ll U_{c}(x)$, where $U_{c}$ is the potential of an isolated streamer of length $l$ :

$$
U_{c}(x)=U_{a}-\int_{R_{a}}^{R_{a}+x} E_{c}\left(x^{\prime}\right) \mathrm{d} x^{\prime}
$$

$U_{a}$ being an anode potential. For $U_{c}=100 \mathrm{kV}, r_{c}=$ $0.01 \mathrm{~cm}$ and $\ln \left(l / r_{c}\right) \approx 10$, we have $E_{r} \simeq 10^{6} \mathrm{~V} \mathrm{~cm}^{-1}$. In this field there can exist the radial ionization wave which causes the channel expansion. Subsequently the radial wave comes to a halt because $E_{r}$ is inversely related to $r_{c}$ and the new radius of the channel is much more than that of the initial avalanche. Our upper estimate for the radius of the isolated streamer in air is about $0.2 \mathrm{~cm}$, whereas it is generally believed to be about $0.01 \mathrm{~cm}[11,12]$.

It is of interest to consider the sensitivity of the results of the $1.5 \mathrm{D}$ simulation of long streamers to the rate coefficients. We performed calculations of the 


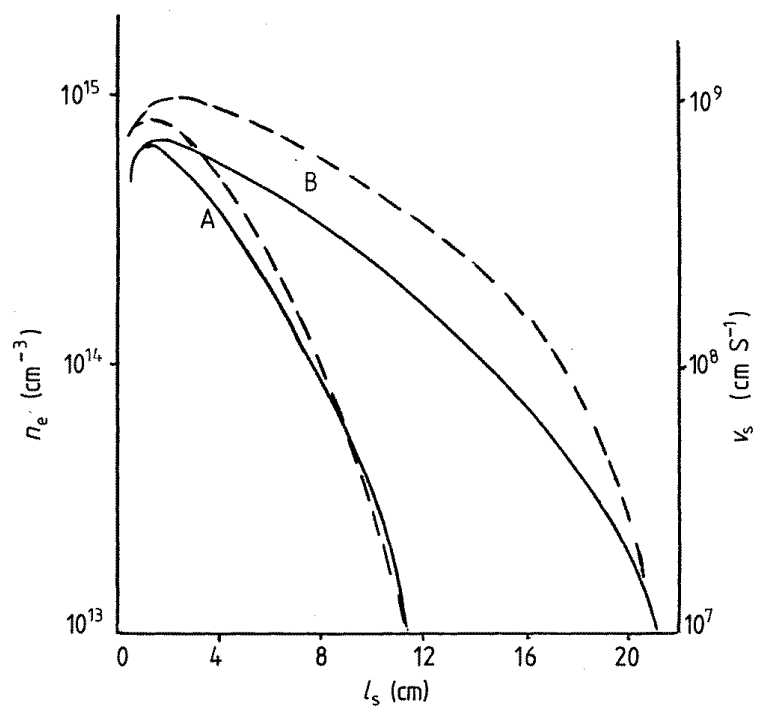

Figure 3. The maximal electron density in the streamer channel (broken curves) and the streamer velocity (full curves) calculated for the simple ionic kinetics with $\beta_{e i}=2 \times 10^{-6} \mathrm{~cm}^{3} \mathrm{~s}^{-1}$ (curve A) and $2 \times 10^{-7} \mathrm{~cm}^{3} \mathrm{~s}^{-1}$ (curve B). The curves correspond to the same initial conditions as those given in figure 1 .

long-streamer properties with a variety of electron rate coefficients described elsewhere [11]. For a spherical anode of $R_{a}=12.5 \mathrm{~cm}$ and the applied voltage of $500 \mathrm{kV}$, the variation in the maximum streamer velocity was less than $25 \%$, that in the maximum electron density was less than $45 \%$ and that in the streamer length was less than $10 \%$. These variations decrease together with a decrease in the anode radius and, for $R_{a}=1 \mathrm{~cm}$, were less than $10 \%$ [11].

Another behaviour is observed when 1.5D simulation of the long streamer assumes the simple ionic kinetics of the streamer plasma. We calculated the long-streamer properties for a three-component plasma composed of electrons, positive and negative ions accounting for direct ionization by electron impact, electron attachment and detachment processes, and electron-ion and ion-ion recombinations with some average rate coefficients. For the electron-ion dissociative recombination we used the rate corresponding to simple ions

$$
\left(\mathrm{O}_{2}^{+}, \mathrm{N}_{2}^{+}\right)\left(\beta_{e i}=2 \times 10^{-7} \mathrm{~cm}^{3} \mathrm{~s}^{-1}\right)
$$

or complex ions

$$
\left(\mathrm{O}_{4}^{+}, \mathrm{N}_{4}^{+}\right)\left(\beta_{e i}=2 \times 10^{-6} \mathrm{~cm}^{3} \mathrm{~s}^{-1}\right) .
$$

This variation in the electron-ion recombination rate has a strong effect on the streamer behaviour. Figure 3 shows that the limiting streamer length varies by a factor of two, whereas, under the same conditions, the maximum electron density varies by a factor of 15 .

The failure to describe the long-streamer dynamics with the simplified kinetics may be explained in terms of a change in the plasma composition during propagation of the streamer. This is evident from figure 4 which shows the calculated positive-ion composition of the streamer plasma versus time near the anode and at a distance of $5.1 \mathrm{~cm}$ from it. $\mathrm{A} \mathrm{N}_{2}^{+}$ion produced by electron impact ionization at the streamer head rapidly reacts with $\mathrm{N}_{2}$ in the three-body reaction

$$
\mathrm{N}_{2}^{+}+\mathrm{N}_{2}+\mathrm{M} \rightarrow \mathrm{N}_{4}^{+}+\mathrm{M}
$$

then, the $\mathrm{O}_{2}^{+}$ion is formed in the charge exchange reaction

$$
\mathrm{N}_{4}^{+}+\mathrm{O}_{2} \rightarrow 2 \mathrm{~N}_{2}+\mathrm{O}_{2}^{+}
$$

and it is removed in the three-body reaction

$$
\mathrm{O}_{2}^{+}+\mathrm{O}_{2}+\mathrm{M} \rightarrow \mathrm{O}_{4}^{+}+\mathrm{M}
$$

Ion-molecule reactions changing the ionic species result in a marked variation in the average rate of the electronion recombination. Therefore, there is reason to think that a simplification of the positive-ion kinetics can result in large errors in the calculation not only of the composition of streamer plasma but also of the average streamer properties, namely the velocity of propagation, the conductivity and the limiting streamer length.

The above arguments hold true for the negative-ion kinetics because of the electron attachment-detachment reactions. Our calculations show that the dominant negative ion is $\mathrm{O}^{-}$at the streamer head and $\mathrm{O}_{2}^{-}$in the channel. These ions are formed in the dissociative electron attachment

$$
\mathrm{e}+\mathrm{O}_{2} \rightarrow \mathrm{O}^{-}+\mathrm{O}
$$

and in the three-body electron attachment

$$
\mathrm{e}+2 \mathrm{O}_{2} \rightarrow \mathrm{O}_{2}^{-}+\mathrm{O}_{2}
$$

respectively, and are removed in the ternary ion-ion recombination

$$
\mathrm{A}^{-}+\mathrm{B}^{+}+\mathrm{C} \rightarrow \mathrm{A}+\mathrm{B}+\mathrm{C}
$$

and electron-detachment reactions in collision with active particles such as $\mathrm{O}$ atoms and excited molecules

$$
\begin{gathered}
\mathrm{A}^{-}+\mathrm{B}^{*} \rightarrow \mathrm{e}+\mathrm{AB} \\
\mathrm{A}^{-}+\mathrm{B}^{*} \rightarrow \mathrm{e}+\mathrm{A}+\mathrm{B} .
\end{gathered}
$$

Figure 5 shows the temporal evolution of the calculated active-particle composition near the anode and at a distance of $5.1 \mathrm{~cm}$ from it. The density of active particles decreases with distance from the anode because near the anode the field decays more slowly with time than that at a considerable distance from it. Although the main mechanism of the negative-ion loss in the streamer channel is the ion-ion recombination, the electron detachment can be important under different conditions. Lowke has proposed that the $\mathrm{O}_{2}\left(\mathrm{a}^{1} \Delta_{g}\right)$ molecule plays a dominant role in electron detachment from $\mathrm{O}_{2}^{-}$ions in the streamer channel [30], whereas our calculation shows that the electron detachment is dominated by the $\mathrm{O}$ atoms. 

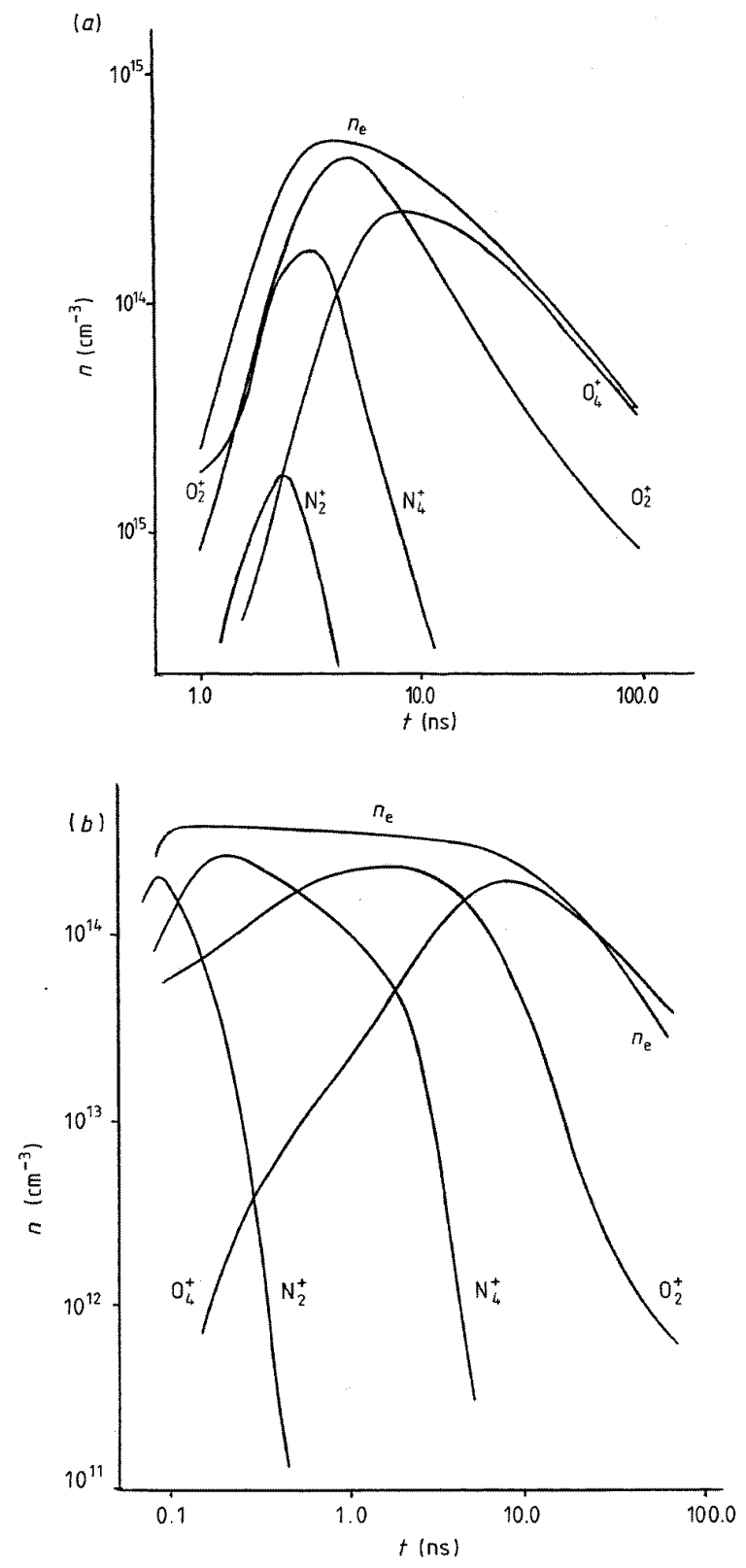

Figure 4. The time evolution of densities of electrons, $\mathrm{O}_{2}^{+}$, $\mathrm{O}_{4}^{+}, \mathrm{N}_{2}^{+}$and $\mathrm{N}_{4}^{+}$in the streamer plasma (a) near the anode and $(b)$ at a distance of $5.1 \mathrm{~cm}$ from it. The curves correspond to the same initial conditions as those given in figure 1.

\section{The model of a streamer with expanded channel}

To account for the ionization expansion of the streamer channel we modified our model which allowed us to make the next step towards a 2D model of the long streamer. The channel radius $r_{c}(x)$ was assumed to be variable and equal to that of the cylinder ionization wave in the radial electric field, where the $x$ axis is directed along the streamer. We performed an auxiliary simulation of ionization wave propagation from the long cylinder electrode and calculated the wave velocity $v_{r}(x)$ and the electron density behind the
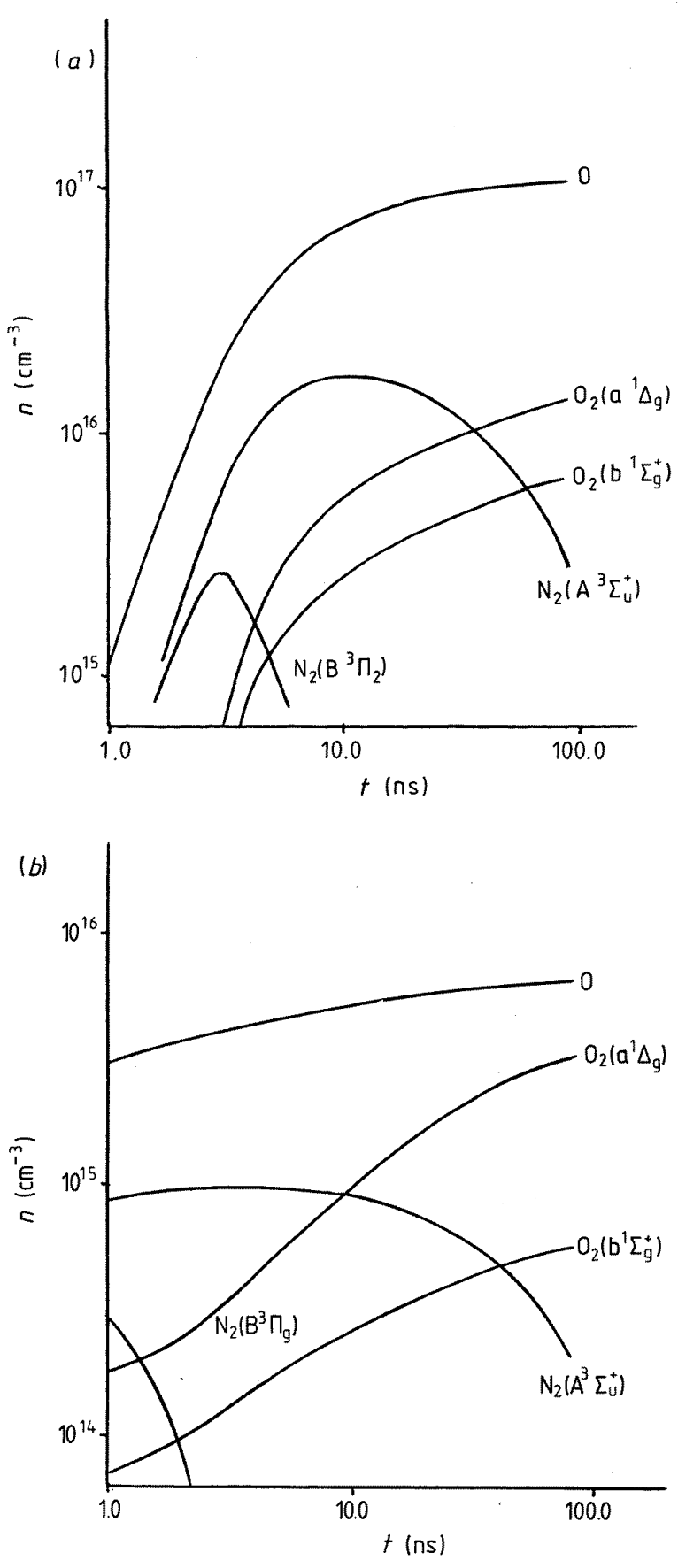

Figure 5. The time evolution of densities of $\mathrm{O}, \mathrm{O}_{2}\left(\mathrm{a}^{1} \Delta_{g}\right)$, $\mathrm{O}_{2}\left(\mathrm{~b}^{1} \Sigma_{g}^{+}\right), \mathrm{N}_{2}\left(\mathrm{~A}^{3} \Sigma_{u}^{+}\right)$and $\mathrm{N}_{2}\left(\mathrm{~B}^{3} \Pi_{g}\right)$ in the streamer plasma (a) near the anode and $(b)$ at a distance of $5.1 \mathrm{~cm}$ from it. The curves correspond to the same initial conditions as those given in figure 1 .

wavefront as functions of $r_{c}$, and the radial electric field $E_{r}\left(x, r_{c}\right)$ in front of the wave where it has a maximum.

To simulate the dynamics of the streamer with an expanded channel, $E_{r}\left(x, r_{c}\right)$ was calculated at each time step $\Delta t$; then, for each interval $\left[x_{k-1}, x_{k}\right]$ the change in radius $\Delta r(x)=v_{r}(x) \Delta t$ and density of electrons in a new plasma layer were obtained from the results of our auxiliary simulation. To calculate a new linear charge density $\tau_{c}(x)$ 
and a new longitudinal electric field the charged-particle densities averaged over a cross section were used. The method of computing this field and the reactions considered are identical to those given in the preceding section.

The radial channel expansion is assumed to stop when the electron density behind the radial ionization wave falls below $5 \times 10^{11} \mathrm{~cm}^{-3}$. In this model the initial radius of the streamer channel, $r_{0}$, is also unknown. However, it will be shown in the following section that the results of this simulation are far less dependent on $r_{0}$ than are those of the $1.5 \mathrm{D}$ simulation in which the channel radius is assumed to be constant.

\section{Simulation of the streamer with an expanded channel in a non-uniform field}

To illustrate the potential of our model of a streamer with an expanded channel, we simulated the propagation of a streamer in air under standard conditions from an anode with $R_{a}=12.5 \mathrm{~cm}$ and with the initial electric field of $40 \mathrm{kV} \mathrm{cm}{ }^{-1}$ near the anode. This corresponds to the conditions of numerous experiments which have been carried out for measuring some averaged long-streamer characteristics, namely total anode current and the length of the streamer propagation $[8,29]$. For $r_{0}=0.1 \mathrm{~cm}$ and $0.02 \mathrm{~cm}$, table 2 shows the calculated streamer properties at the instant at which the total anode current has a maximum, $i_{a \text { max }}$. Here $t_{\text {max }}$ is the period for the current enhancement up to $i_{a \text { max }}, l_{s}$ and $v_{s}$ are the streamer length and velocity at that instant, respectively, $r_{\max }$ is the maximum radius of the channel and $n_{e}$ is the electron density for the cross section at $r_{c}=r_{\max }$. The five-fold decrease in $r_{0}$ causes the results of this simulation to vary by $20-40 \%$ (for $n_{e}$ ), whereas those of the $1.5 \mathrm{D}$ simulation in which the channel radius is fixed vary by a factor of ten.

Our simulation of the streamer with an expanded channel is consistent with the results of a number of experiments $[8,29]$. For the amplitude of the pulse of the total anode current the difference between the theory and the measured magnitude (10 A) is about $10 \%$, whereas the 1.5D simulation gives the hundreds of percent difference which cannot be diminished. The measured magnitude of $l_{s}$ is $65 \mathrm{~cm}$ [31], which is also in good agreement with the result of our calculations yielding $70 \mathrm{~cm}$.

Figure 6 shows the calculated axial profiles of $r_{c}$, electron density averaged over the cross section, and axial electric field $E_{c}$ at the peak value of the total anode current. The radius $r_{c}$ grows with the distance from the anode and has a maximum of $0.45 \mathrm{~cm}$ at $x=13.5 \mathrm{~cm}$. At greater distances from the anode $r_{c}$ steeply falls to the initial channel radius of $0.1 \mathrm{~cm}$. The axial profile of $r_{c}$ near the anode is usually affected by the anode-surface shielding which leads to a sharp fall in the space charge at small $x$ as is shown in figure 6. Under these conditions $r_{c}$ is small and constant along the streamer because the radial electric field tends to zero in the immediate vicinity of any metallic surface. Our calculation shows that the conduction current varies slightly with $x$ not far from the anode. Therefore, current conservation requires a steep increase in $E_{c}$ and $n_{e}$ near the anode owing to a decrease in $r_{c}$.

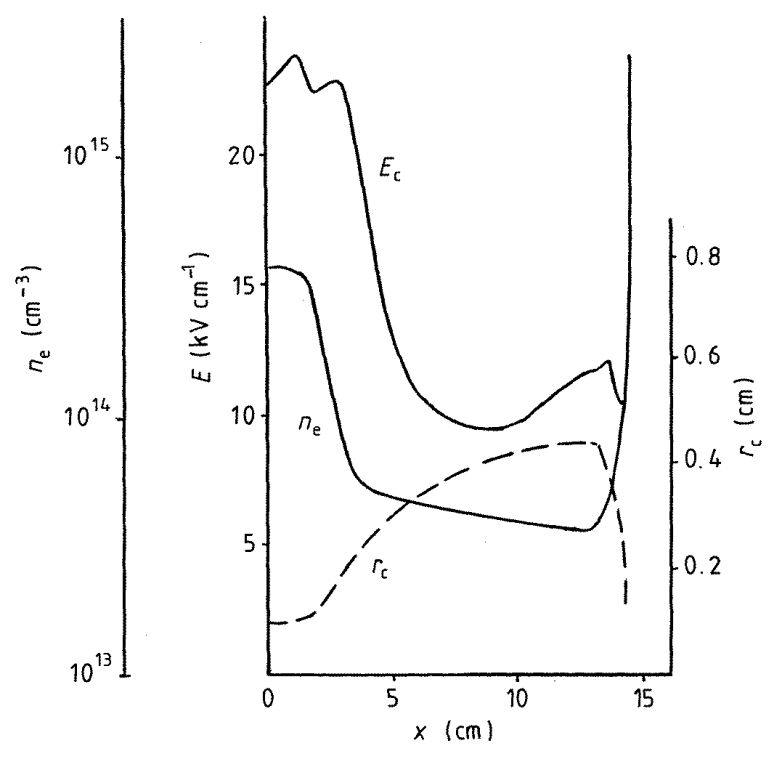

Figure 6. Axial profiles of $r_{c}, n_{e}$ and $E_{c}$ at the instant at which the total anode current peaks $(t=39.3 \mathrm{~ns})$. Initial conditions used were a spherical anode with $R_{a}=12.5 \mathrm{~cm}$ and applied electric field strength near the anode of $40 \mathrm{kV}$ $\mathrm{cm}^{-1}$.

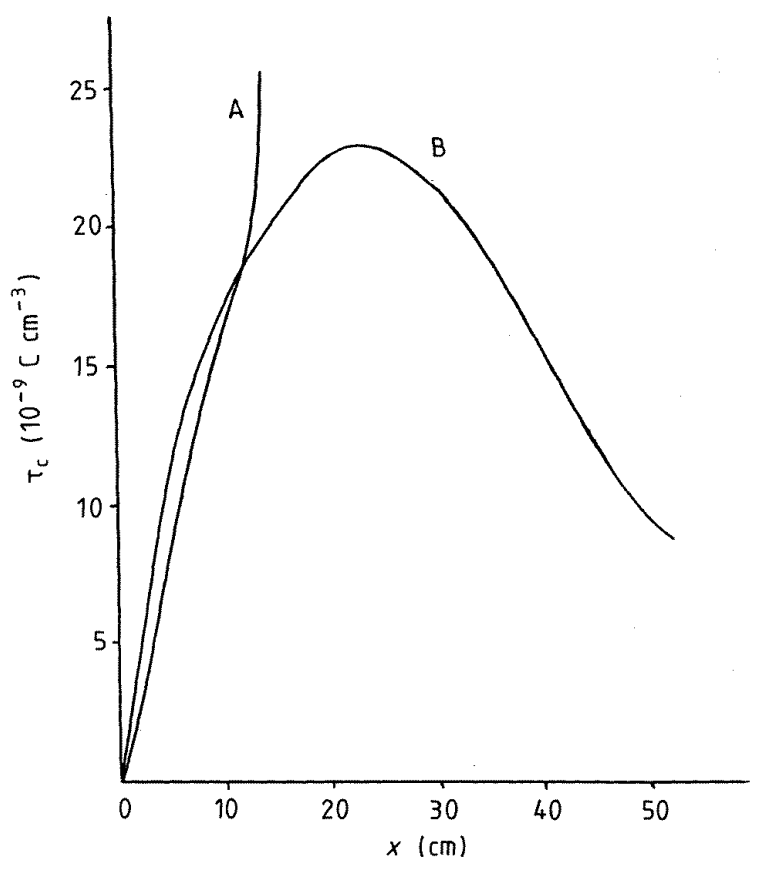

Figure 7. Axial profiles of $\tau_{c}$ at $39.3 \mathrm{~ns}$ (curve A) and 138 ns (curve $B$ ). The curves correspond to the same initial conditions as those given in figure 6 .

In our simulations we observed a strong $r_{0}$-dependence of the electron density at small $x, n_{e a}$. A five-fold increase in $r_{0}$ resulted in a 30 -fold decrease in $n_{e a}$, namely $n_{e a} \simeq$ $r_{0}^{-2}$, because the axial electric field near the anode is almost independent of $r_{0}$. Clearly our approach fails to determine $n_{e a}$ and $r_{0}$ independently. 
Table 2. The streamer parameters corresponding to the maximal value of the total anode current, $i_{a \max }$.

\begin{tabular}{lllllll}
\hline$r_{0}(\mathrm{~cm})$ & $t_{\max }\left(10^{-8} \mathrm{~s}\right)$ & $l_{s}(\mathrm{~cm})$ & $i_{\max }(\mathrm{A})$ & $v_{s}\left(10^{9} \mathrm{~cm} \mathrm{~s}^{-1}\right)$ & $r_{\max }(\mathrm{cm})$ & $n_{e}\left(10^{13} \mathrm{~cm}^{-3}\right)$ \\
\hline 0.1 & 4 & $\simeq 15$ & 11.0 & 1.2 & 0.45 & 3.5 \\
0.02 & 3 & $\simeq 15$ & 8.9 & 1 & 0.37 & 2.5 \\
\hline
\end{tabular}

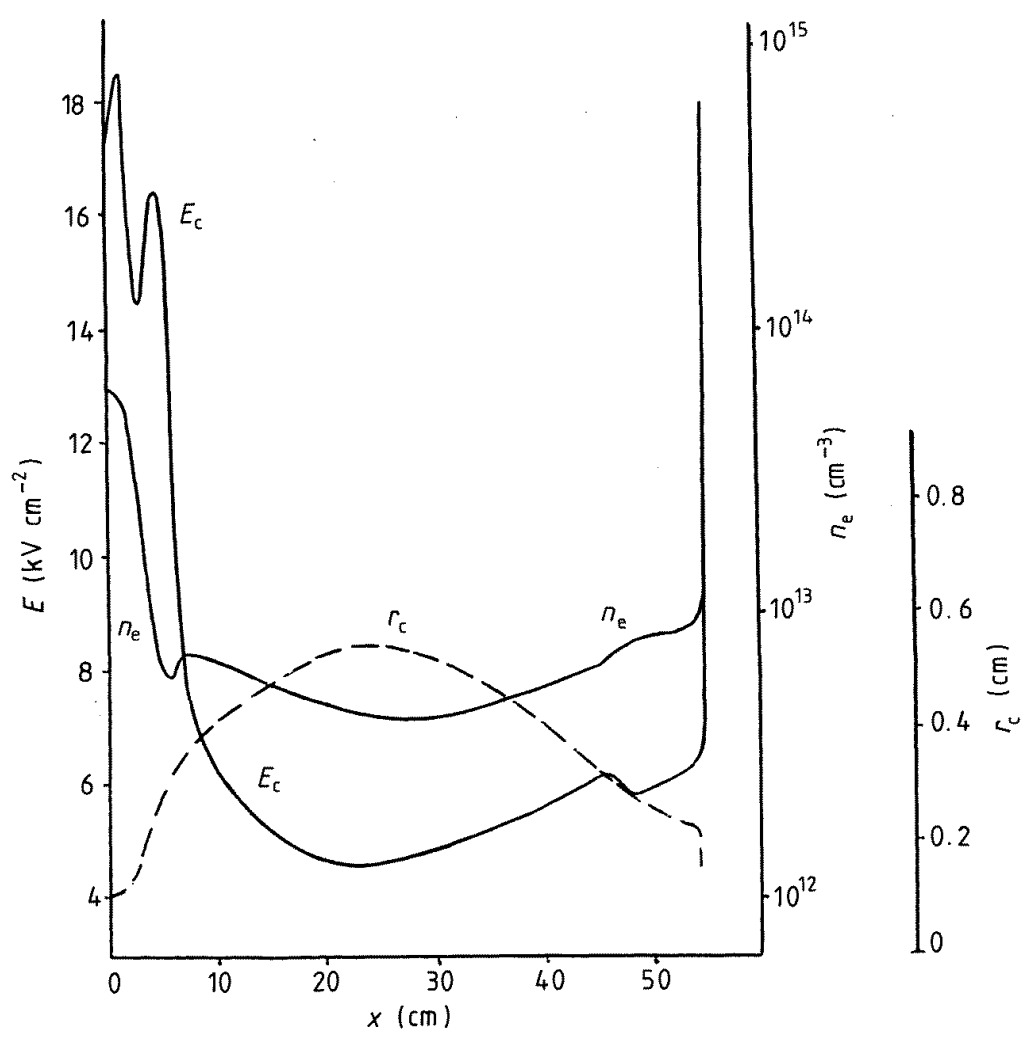

Figure 8. Axial profiles of $r_{c}, E_{c}$ and $n_{e}$ at $138 \mathrm{~ns}$. The curves correspond to the same initial conditions as those given in figure 6 .

For a sufficiently long streamer, the axial profile of the linear charge density $\tau_{c}$ has a peak owing to a voltage drop along the channel; this is shown in figure 7 . Figure 8 shows that $r_{c}$ also has a maximum at the same distance from the anode whereas $E_{c}$ and $n_{e}$ have minima, $E_{c \text { min }}$ and $n_{\text {emin }}$. For a period of $0.25-0.3 \mu \mathrm{s}, n_{e \text { min }}$ drops by a factor of 100 because of electron attachment and electron-ion recombination. In contrast to electron density and conductivity, the axial electric field is almost constant in the channel away from the anode and the head of a long streamer. The average magnitude of $E_{c}$ is about $5.3-5.4 \mathrm{kV} \mathrm{cm}{ }^{-1}$ for a streamer of more than $50 \mathrm{~cm}$ in length. This agrees well with numerous measurements of the average electric field in a streamer after bridging long air gaps at atmospheric pressure, which yield about $5 \mathrm{kV} \mathrm{cm}^{-1}$ [8-10].

\section{Simulation of the streamer with an expanded channel in a uniform field}

In most of the theoretical studies the propagation of the streamer is considered in uniform external fields. (The
Table 3. The steady propagation velocity of the long streamer in the uniform field of $12 \mathrm{kV} \mathrm{cm}^{-1}$ versus $r_{c}$.

\begin{tabular}{lllllc}
\hline$r_{c}(\mathrm{~cm})$ & 0.02 & 0.03 & 0.05 & 0.06 & 0.08 \\
$v_{s}\left(10^{8} \mathrm{~cm} \mathrm{~s}^{-1}\right)$ & 3.0 & 4.0 & 6.5 & 8.0 & 12.0 \\
\hline
\end{tabular}

external field is the field in the gap before the streamer initiation.) There is reason to think that this occurs for corona streamers in front of a leader [32]. We also simulated the propagation of a long streamer in a uniform field and showed that the streamer can propagate at a constant velocity with a steady profile of electron density at the head. As before, the results of the 1.5D simulation in the fixed-channel-radius approximation depend strongly on $r_{c}$. Table 3 presents the velocity of the steady propagation of the streamer in a uniform field of $12 \mathrm{kV} \mathrm{cm}^{-1}$ as a function of $r_{c}$.

In what follows we use the model of the streamer with an expanded channel. The streamer is assumed to start from a spherical anode with $R_{a}=0.2 \mathrm{~cm}$ at the anode potential of $U_{a}=10 \mathrm{kV}$ and to propagate in an additional 


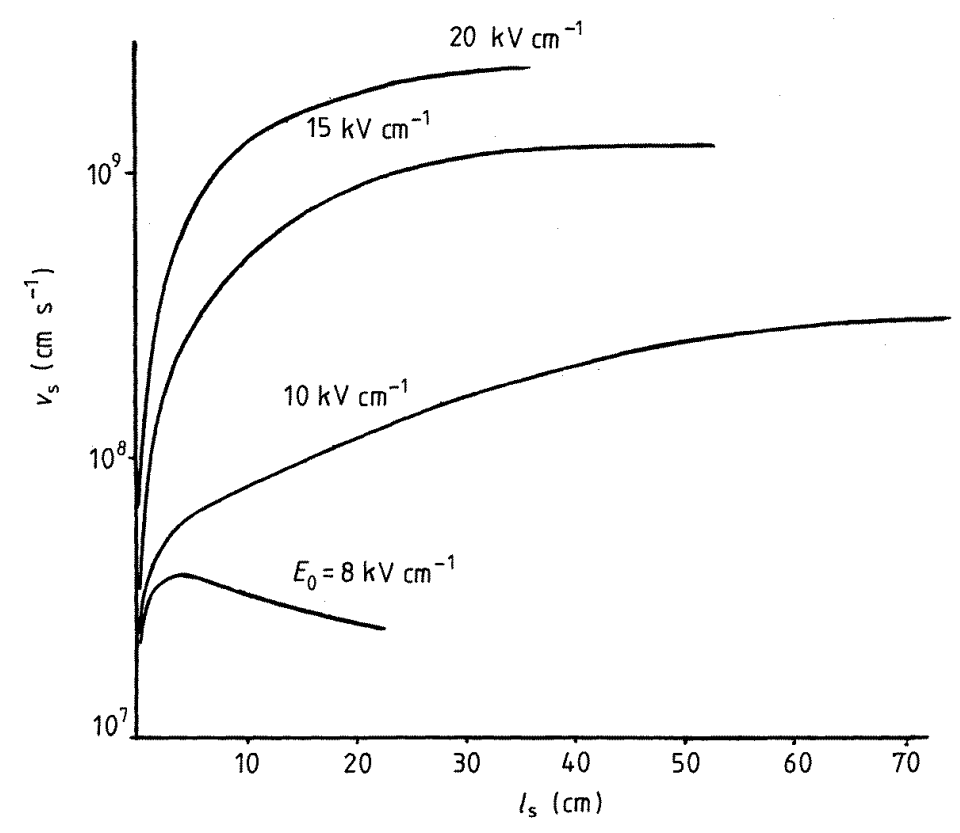

Figure 9. $v_{c}$ versus the streamer length at different values of $E_{0}$.

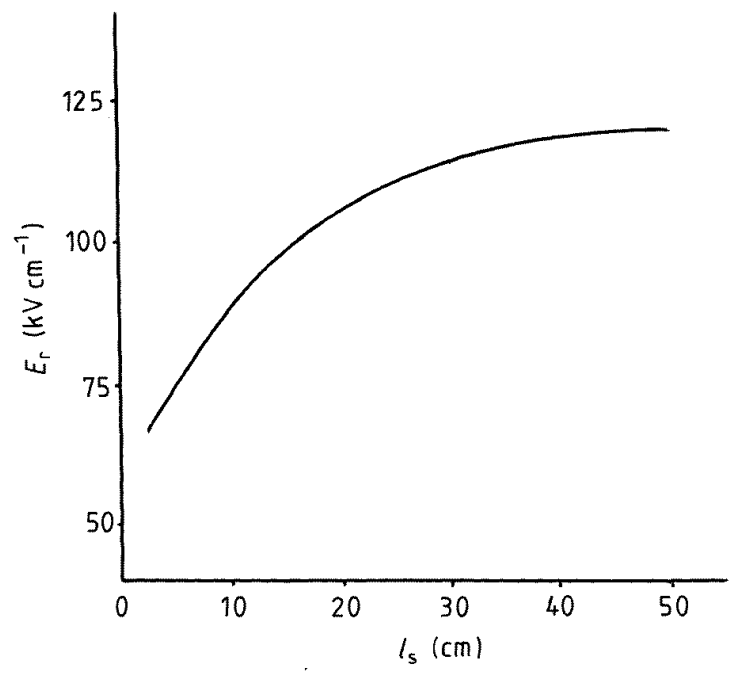

Figure 10. The peak electric field at the streamer head versus the streamer length at $E_{0}=15 \mathrm{kV} \mathrm{cm}^{-1}$.

uniform electric field $E_{0}$. Once the length of the streamer has attained $2 \mathrm{~cm}$, the anode field in front of the ionization wave becomes less than $0.5 \mathrm{kV} \mathrm{cm}^{-1}$; that is, the effect of this field on further streamer propagation can be neglected.

Our simulation predicts a steady propagation of the streamer in an infinite gap between anode and cathode when $E_{0}$ is higher than the threshold $E_{\text {min }}=8-8.5 \mathrm{kV} \mathrm{cm}^{-1}$. The field $E_{\min }$ slightly depends on $r_{0}, R_{a}$ and $U_{a}$. For instance, a $50 \%$ variation in any one of the last quantities results in $10 \%$ variation in $E_{\min }$. $E_{\min }$ calculated in this work agrees qualitatively with data obtained in experiments with steeply non-uniform fields which demonstrate streamer bridging of the atmospheric-air gaps from $0.1-10 \mathrm{~m}$ in length whereas the averaged electric field exceeds $5 \mathrm{kV} \mathrm{cm}^{-1}$ [8-10]. We have not succeeded in diminishing the difference between calculated and experimental $E_{\min }$ by varying the rate coefficients of processes considered. This difference can be ascribed to streamer branching, the field non-uniformity and the unsteady regime of streamer propagation in the experiments.

Figure 9 shows the streamer velocity in the uniform electric field versus the streamer length. $E_{0}$ has a dramatic effect on the long-streamer behaviour. For $E_{0}<E_{\text {min }}$, the streamer is accelerated over a distance of several centimetres and subsequently decelerated with a rate which depends on $E_{0}$. If $E_{0}$ approaches $E_{\min }$, then the streamer can propagate up to tens of centimetres. When $E_{0}>E_{\text {min }}$, the streamer propagation becomes steady; in this case the time and length of the streamer relaxation decrease with increasing $E_{0}$. The least velocity of steady propagation which is observed at $E_{0}=E_{\text {min }}$ and equals $(3-4) \times$ $10^{7} \mathrm{~cm} \mathrm{~s}^{-1}$ qualitatively correlates with the available experimental data (about $(1-2) \times 10^{7} \mathrm{~cm} \mathrm{~s}^{-1}$ ) [33].

The steady-state propagation of the streamer is observed when the electric field at its head becomes steady, as is shown in figure 10. An increment in the streamer-head field is induced by a polarization charge in the streamer channel in the external field. At the infinite conductivity of the channel plasma the space charge would increase indefinitely with the streamer length, whereas in reality it is limited by the voltage drop along the finite-conductivity plasma channel. The profiles of charge and field at the streamer head become steady when this voltage drop, $U_{c}$, obeys the equation

$$
-\frac{\mathrm{d} U_{c}}{\mathrm{~d} x}=E_{c} \approx E_{0}
$$

that is, under steady-state conditions the longitudinal electric field $E_{c}$ is close to $E_{0}$ in the channel plasma 


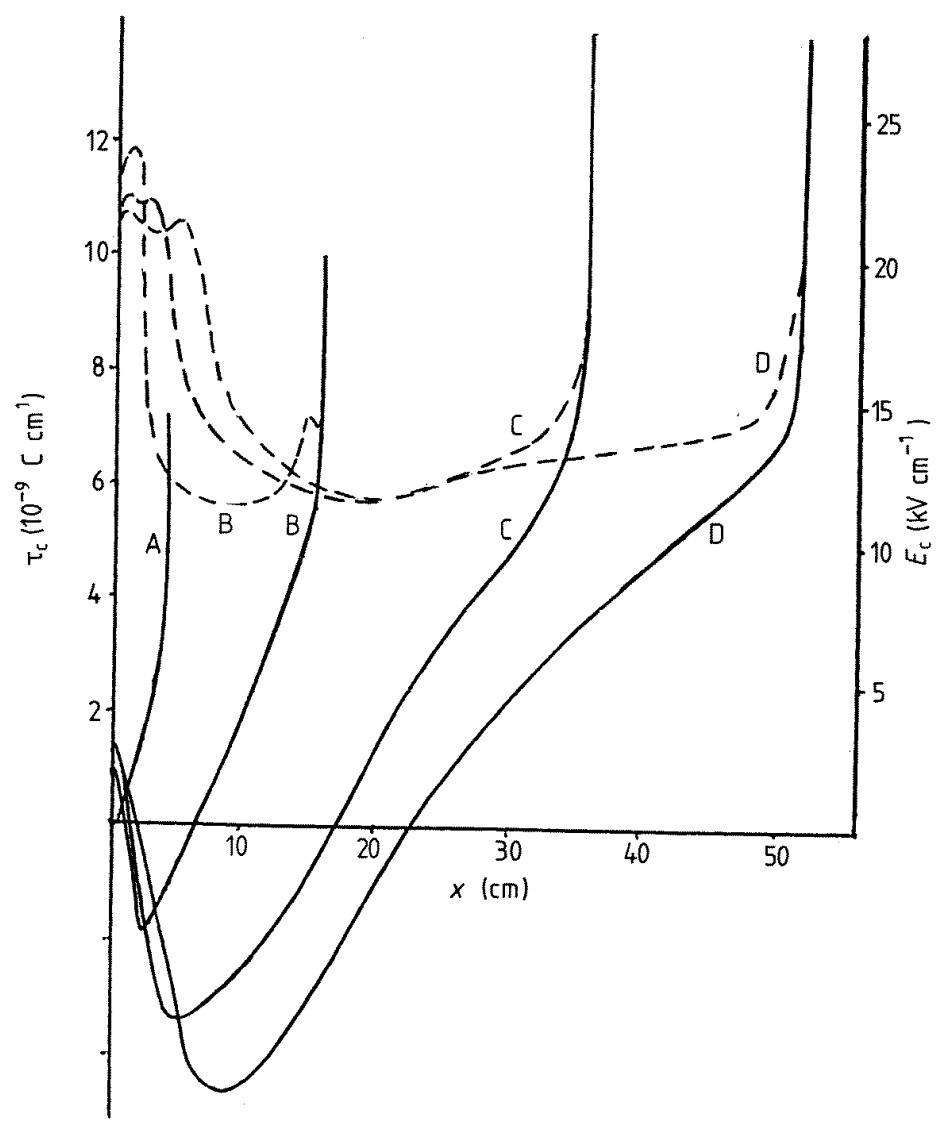

Figure 11. Axial profiles of $\tau_{c}$ (full curves) and $E_{c}$ (broken curves) for $E_{0}=15 \mathrm{kV} \mathrm{cm}^{-1}$ at $40 \mathrm{~ns}$ (curve A), $65 \mathrm{~ns}$ (curve B), 85 ns (curve C) and 98 ns (curve D).

Table 4. The length-averaged longitudinal field in the channel of the steady streamer versus $E_{0}$.

\begin{tabular}{lllll}
\hline$E_{0}\left(\mathrm{kV} \mathrm{cm}^{-1}\right)$ & 8 & 10 & 15 & 20 \\
$E_{c} / E_{0}$ & 0.96 & 0.98 & 0.96 & 0.94 \\
\hline
\end{tabular}

everywhere but near the anode and at the streamer head. This agrees well with curves shown in figure 11 and with the length-averaged channel fields given in table 4 as a function of $E_{0}$.

Figure 12 shows the axial profiles of the electron density averaged over the cross section at $E_{0}=10$ and $15 \mathrm{kV} \mathrm{cm}^{-1}$. Under the conditions considered these profiles and the conduction-current profiles given in figure 13 become strongly non-uniform. The current gradient steepens with decreasing $E_{0}$ at a given streamer length because an increment in the lifetime of the streamer leads to decay of the 'old' plasma segments through electron attachment and electron-ion recombination.

Except very close to the anode, the streamer current drops as $x$ decreases; that is, the external circuit fails to supply new segments of the streamer with electric charge because of the low conductivity of the decaying plasma away from the streamer head where the electron current directed at the anode decreases to form negative space charge. Here the low conductivity of the plasma prevents this charge going to the anode. Figure 11 shows that the charge profile becomes bipolar: the domain of the channel near the anode becomes negatively charged. For a sufficiently long streamer this positive charge has a steady distribution in a moving frame of reference and propagates with the streamer velocity whereas the negativecharge tail increases further. Qualitatively, the distribution of the space charge in the streamer channel is similar to that of the polarization charge in a moving, perfectly conducting plasma with some distortion because of the weak connection to the anode. At the streamer head the profiles of the plasma-component densities and electric field also become steady; therefore, this agrees with the hypothesis by Bazelyan et al [34] that the steady-state propagation of a streamer can be treated as a propagation of the plasma domain of fixed length in an external electric field.

Figure 14 shows the steady axial profile of $r_{c}$ at $E_{0}=15 \mathrm{kV} \mathrm{cm}^{-1}$. We see that the channel radius sharply changes in the streamer head. This phenomenon could be observed experimentally by means of standard photography to test our hypothesis that the streamer channel expands due to ionization in the radial field.

The steady channel radius $r_{c s}$ depends strongly on $E_{0}$. Table 5 gives $r_{c s}$, the velocity of the steady propagation of the streamer, the averaged electron density at the streamer 


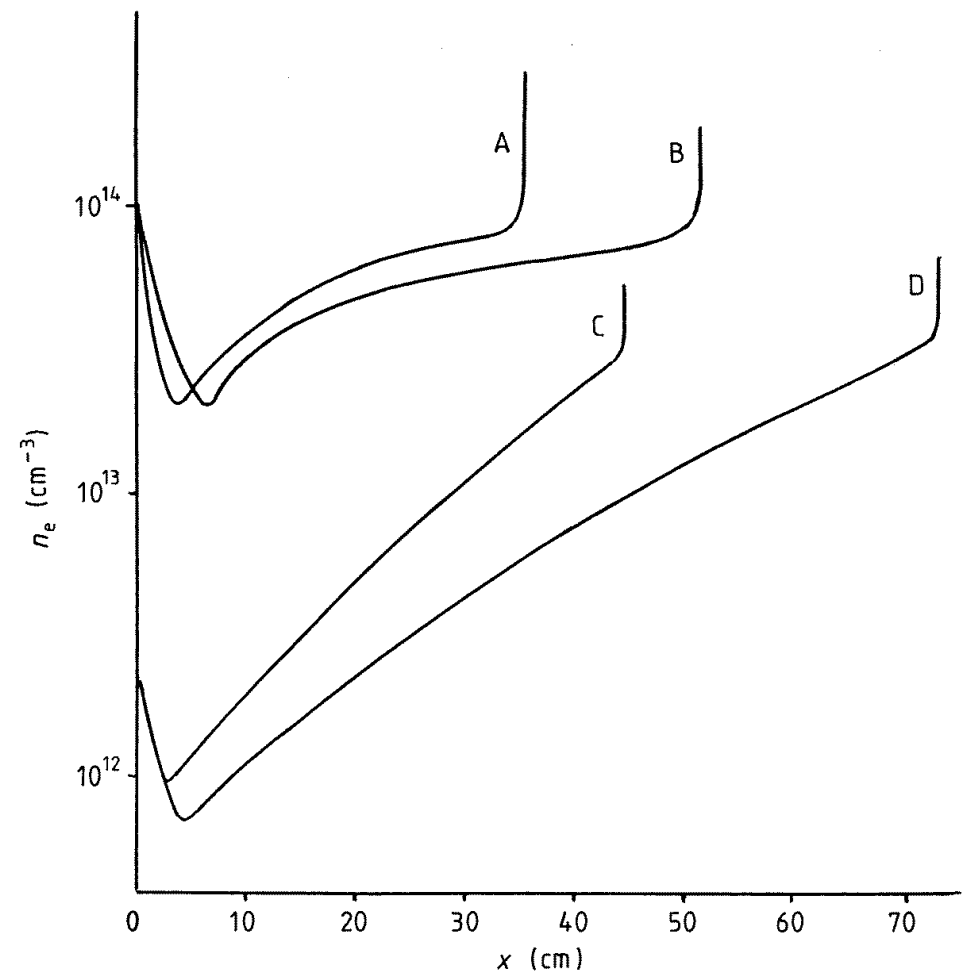

Figure 12. Axial profiles of $n_{e}$ for $E_{0}=15 \mathrm{kV} \mathrm{cm}^{-1}$ at $85 \mathrm{~ns}$ (curve A) and $98 \mathrm{~ns}$ (curve B) and for $E_{0}=10 \mathrm{kV} \mathrm{cm}^{-1}$ at $442 \mathrm{~ns}$ (curve C) and 545 ns (curve D).

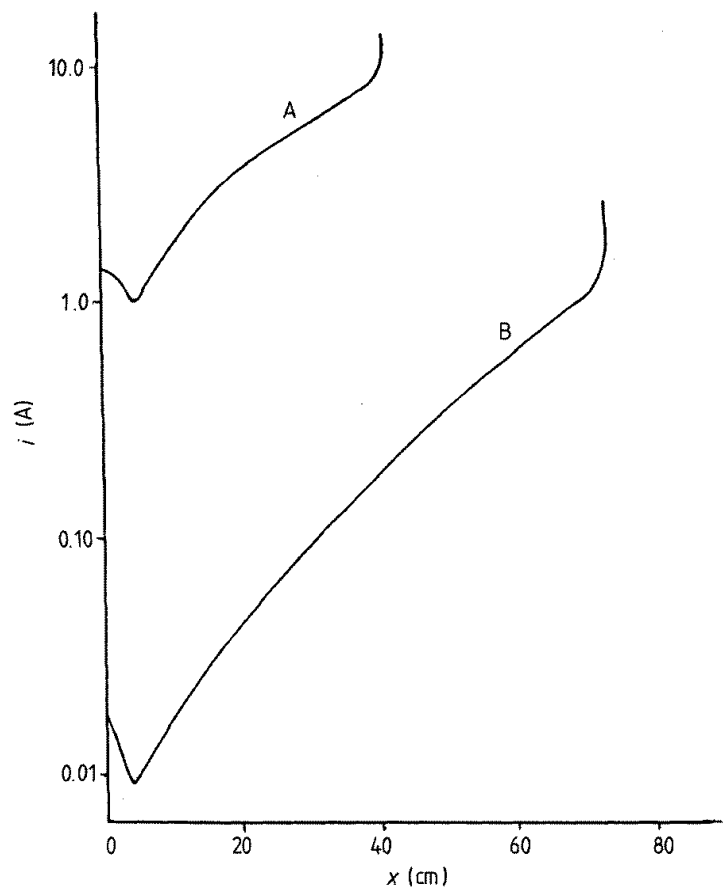

Figure 13. Axial profiles of the conduction current for $E_{0}=10 \mathrm{kV} \mathrm{cm}^{-1}$ at $90.2 \mathrm{~ns}$ (curve A) and for $E_{0}=15 \mathrm{kV}$ $\mathrm{cm}^{-1}$ at $545 \mathrm{~ns}$ (curve B).

head at the section of radius $r_{c s}$ and the density of space charge per unit length at the head versus $E_{0}$. If $E_{0}$

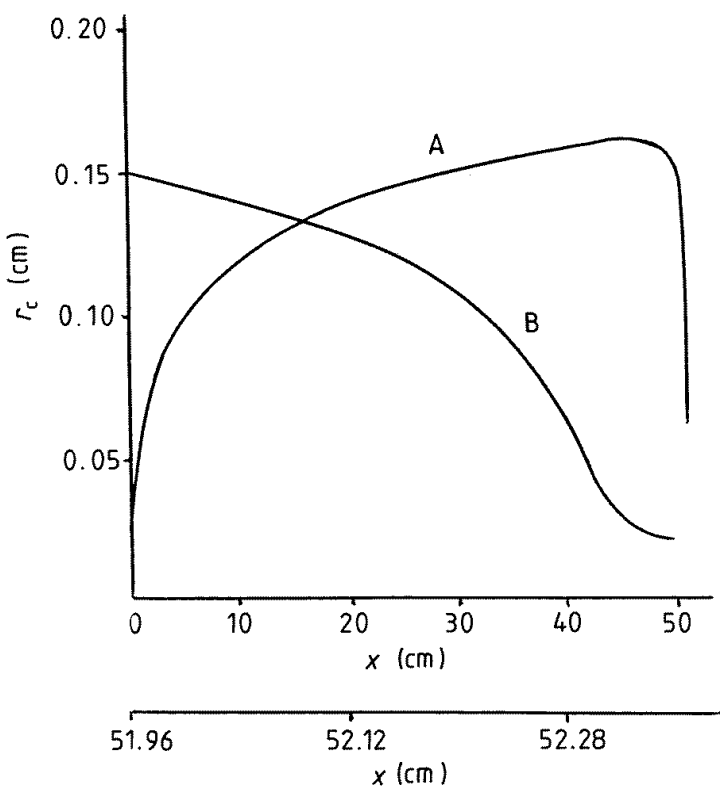

Figure 14. The axial profile of $r_{c}$ for $E_{0}=15 \mathrm{kV} \mathrm{cm}^{-1}$ at 98 ns (curve A, upper abscissa). Curve $B$ and the lower abscissa show a more accurate representation of this profile at the streamer head.

greatly exceeds $E_{\text {min }}$, then $r_{c s}$ is roughly proportional to $E_{0}$, whereas other quantities increase more steeply with $E_{0}$. For instance, $v_{s}$ is roughly proportional to $E_{0}^{3}$. 
Table 5. The steady radius of the streamer channel, the velocity of the steady streamer propagation, the averaged electron density and the density of space charge per unit length near the streamer head versus $E_{0}$.

\begin{tabular}{lllcc}
\hline$E_{0}\left(\mathrm{kV} \mathrm{cm}^{-1}\right)$ & $r_{c s}(\mathrm{~cm})$ & $v_{s}\left(10^{8} \mathrm{~cm} \mathrm{~s}^{-1}\right)$ & $n_{e}\left(10^{13} \mathrm{~cm}^{-3}\right)$ & $\tau_{c}\left(10^{-9} \mathrm{C} \mathrm{cm}^{-1}\right)$ \\
\hline 10 & 0.105 & 3.2 & 4 & 7.7 \\
15 & 0.16 & 13 & 8 & 15 \\
20 & 0.22 & 25 & 10 & 21
\end{tabular}

\section{Discussion}

The ionization expansion of the streamer channel becomes more pronounced as the surface charge of the channel increases. This depends on the amplitude, the pulserise rate of the applied voltage and the voltage drop along the streamer channel. To increase the ionization expansion, it is desirable to attain the voltage amplitude as quickly as possible and consequently to minimize the voltage drop along the channel by decreasing its length. In addition, the axial wavefront should escape the anode region where the surface-bound charge shields the streamer channel and decreases its charge which creates the radial electric field. Therefore, at a given voltage, we have a maximum expansion of the channel provided that the streamer develops from the anode with a minimum radius and the impulse voltage has a minimal risetime.

The anode-radius effect can be detected experimentally. For instance, our calculation for a voltage of $500 \mathrm{kV}$ shows that a change in the radius $R_{a}$ from 12.5 to $5 \mathrm{~cm}$ results in a proportional increase in the channel radius with a maximum of $1.5 \mathrm{~cm}$. On performing similar calculations for nearuniform fields we obtain a maximum channel radius of no more than $0.2 \mathrm{~cm}$. This strong $R_{a}$-dependence of the channel radius in the vicinity of the anode where branching is absent and the streamer path is predictable can be found experimentally to test our hypotheses. This requires static photography of the initial regions of streamers by means of long-focus optics provided a voltage source with short risetime (down to 5-10 ns) is used.

As was noted above, the inclusion of the ionization expansion of the streamer channel into consideration increases the calculated magnitude of the total anode current up to the experimental magnitude. It is of interest to note that the channel expansion has an indirect effect on the anode current because there is no radial electric field and, therefore, no expansion near the anode. The electron current flowing from the expanded regions of the channel accumulates electrons near the anode; this induces an additional axial field over a long period. Our calculation shows that this field has the magnitude of more than $30 \mathrm{kV}$ $\mathrm{cm}^{-1}$ for $20 \mathrm{~ns}$. The additional ionization increasing the electron density by a factor of 3.5 provides a way for the current of about 10 A to flow through the 'bottleneck' of the streamer channel near the anode.

Our study should clarify the question of the steady propagation of a streamer in a uniform field. There are contrary views concerning the long streamer's behaviour. The streamer with the channel considered as a perfect conductor is known to be accelerated permanently [35]. On the other hand, plasma decay in the channel regions away from the streamer head can make the streamer move at a constant velocity [34]. Our calculation shows that the streamer steadily propagates in a uniform field of more than $8 \mathrm{kV} \mathrm{cm}^{-1}$ without any plasma decay. We obtained that, at the first stage as the streamer is accelerated, the longitudinal electric field in the streamer channel increases gradually because the electrical conductivity of the channel increases more slowly than does the current density. At any length of the streamer, $l_{s c r}$, if the voltage along the streamer, $\Delta U_{s}$, achieves the voltage of the external field $E_{0} l_{s c r}$, this field is no longer cancelled in the channel. Then the streamer-plasma polarization which increases the space charge and electric field in the streamer head disappears. Transition to a steady state of the streamer head results in streamer propagation at the constant velocity obtained in our calculation.

As is mentioned above, during the steady motion the average field in the channel is approximately equal to the external field $E_{0}$, whereas the transition to the steady state is not related to a decay of the streamer plasma. It can be shown that this transition takes place even when the channel plasma conductivity does not fall with time. In the approximation of a fixed channel radius we obtain from equation (7) that $\sigma_{s} \simeq n_{e} \simeq v_{s}$, where $\sigma_{s}$ is the streamer plasma conductivity. Using the relation $j \simeq v_{s} c_{s} \varphi_{s}\left(c_{s}\right.$ and $\varphi_{s}$ are the average capacitance and potential of the channel, respectively) we have that $E_{c}=j / \sigma_{s} \simeq \varphi_{s}$; that is, the electric field in the channel does not depend on the density of electrons and increases monotonically as the field at the head and $\varphi_{c}$ rise. The streamer accelerates until $E_{c}$ reaches $E_{0}$. Thereafter all the quantities characterizing the streamer head, including $v_{s}$, become time-independent; that is, in our calculations we obtain steady streamer propagation. The higher $E_{0}$, the higher the current and $\varphi_{s}$ corresponding to the transition to steady streamer propagation. For instance, when $E_{0}$ varies from 10 to $20 \mathrm{kV} \mathrm{cm}{ }^{-1}$, the calculated conduction anode current increases from 0.02 to $10 \mathrm{~A}$ for the time at which the streamer velocity becomes steady.

\section{Conclusions}

Our numerical simulation of the long-streamer propagation in air assuming a fixed radius of the streamer channel shows a strong dependence of the streamer properties on the radius and the considered model of charge-particle kinetics. Taking into account the ionization expansion of the streamer channel, we obtained results slightly dependent on the initial channel radius. The simulation of the streamer propagation from a spherical anode in a nonuniform electric field agrees well with the results of a lot of experiments in which the total anode current, the streamer 
length and the average electric field in the streamer channel were measured. For the streamer evolution in a uniform electric field, the calculation yields a steady propagation of the streamer at a strong field and a strong field-dependence of the streamer properties. In this case, the space charge distribution in the streamer channel is similar to that of the polarization charge in a moving perfectly conducting plasma disturbed by a weak connection to the anode.

\section{Acknowledgments}

This work was supported by the Russian Foundation of Fundamental Investigations under Contract 95-02-03944-a and by the Department of Electric Power Problems of the Russian Academy of Science.

\section{References}

[1] Bazelyan E M, Goncharov V N and Gorunov A Yu 1985 Izv. Acad. Nauk SSSR OTN 2154

[2] Bazelyan E M 1982 Izv. Acad. Nauk SSSR OTN 382

[3] Clements J S, Mizuno A, Finney W C and Davis R H 1986 IEEE Trans. Indust. Appl. 21183

[4] Masuda S 1988 Pure Appl. Chem. 60727

[5] Masuda S and Nakao H 1990 IEEE Trans. Indust. Appl. 26 374

[6] Lesaint O and Gournay P 1994 J. Phys. D: Appl. Phys. 27 2111

[7] Gallimberti J 1979 J. Physique 40 C7-193

[8] Les Renardières Group 1977 Electra $\mathbf{5 3} 31$

[9] Meek J M and Craggs J D 1978 Electrical Breakdown of Gases (New York: Wiley)

[10] Bazelyan E M and Razhanskii I M 1988 Spark Discharge in Air (Novosibirsk: Nauka)
[11] Aleksandrov N L, Bazelyan A E, Bazelyan E M and Kochetov I V 1995 Plasma Phys. Rep. 2157

[12] Kunhardt E E 1985 Proc. XVII Int. Conf. on Phenomena in Ionized Gases ed J S Bakos and Z Sorlei (Budapest: KFKI) p 345

[13] Pavlovskii A I, Babich L P, Soboleva T V and Shamraev B N 1982 Dokl. Acad. Nauk SSSR 246840

[14] Bortnik I M, Kochetov I I and Ul'yanov K N 1982 Teplofiz. Vys. Temp. 20193

[15] Kunhardt E E and Tzeng Y 1988 Phys. Rev. A 381410

[16] Shveigert V A 1989 Zh. Prikl. Mekh. Tehn. Fiz. 110

[17] Dhali S K and Williams P F 1985 Phys. Rev. A 311219

[18] Wu C and Kunhardt E E 1988 Phys. Rev. A 374396

[19] Wang M and Kunhardt E 1990 Phys. Rev. A 422366

[20] Jing-Ming Guo and Chwan-Hwa Wu 1993 J. Phys. D: Appl. Phys. 26487

[21] Vitello P A, Penetrante B M and Bardsley J N 1994 Phys. Rev. E 495574

[22] Kulikovsky A A 1994 J. Phys. D: Appl. Phys. 272564

[23] Morrow R 1985 Phys. Rev. A 321799

[24] Gallimberty J 1988 Pure Appl. Chem. 60663

[25] Shveigert V A 1990 Teplofiz. Vys. Temp. 281056

[26] Kossyi J A, Kostinsky A Yu, Matveyev A A and Silakov V P 1992 Plasma Sources Sci. Technol. 1207

[27] Bazelyan A E and Bazelyan E M 1993 High Temp. 31799

[28] Bazelyan A E and Bazelyan E M 1994 High Temp. 32332

[29] Stekolnikov I S 1960 The Nature of the Long Spark (Moscow: Izdat Acad Nauk SSSR); Engl. transl. 1962 USA Department of Commerce FTD-TT-61-149

[30] Lowke J J 1992 J. Phys. D: Appl. Phys. 25202

[31] Brago E N and Stekolnikov I S 1958 Izv. Acad. Nauk SSSR OTN 1151

[32] Petrov N I, Avanskii V R and Bombenkova N V 1994 Zh. Tekh. Fiz. 64 (6) 50

[33] Gorin B N and Shkilev A V 1974 Electrichestvo 229

[34] Bazelyan E M and Gorunov A Yu 1986 Electrichestvo 11 27

[35] Lozanskii E D 1976 Usp. Fiz. Nauk 120 363; 1976 Sov. Phys.-Usp. 19894 